\title{
Klotho gene improves oxidative stress injury after myocardial infarction
}

\author{
ZHUOFAN XU ${ }^{1}$, SHAOXIN ZHENG ${ }^{2}$, XIAOQIAN FENG $^{1}$, CHENGZHE CAI $^{1}$, XIANQU YE $^{1}$ and PINGFANG LIU $^{1}$ \\ ${ }^{1}$ Department of Internal Medicine-Cardiovascular, Guangzhou 12th People's Hospital, Guangzhou, Guangdong 510620; \\ ${ }^{2}$ Department of Internal Medicine-Cardiovascular, Sun Yat-sen Memorial Hospital, \\ Sun Yat-sen University, Guangzhou, Guangdong 510200, P.R. China
}

Received September 26, 2019; Accepted April 7, 2020

DOI: $10.3892 /$ etm.2020.9484

\begin{abstract}
The aim of the present study was to investigate the effects and mechanisms of the Klotho gene in oxidative stress injury after myocardial infarction. Sprague-Dawley rats were divided into five groups (sham, model, pDC316, LY294002, and pDC316-Klotho). Subsequently, the superoxide dismutase (SOD), glutathione (GSH), and malondialdehyde (MDA) concentrations were measured in myocardial tissues. Additionally, pathological differences among the groups were evaluated using hematoxylin and eosin and Masson's trichrome staining. Apoptosis was assayed by terminal deoxynucleotidyl transferase 2'-deoxyuridine-5'-triphosphate nick end-labeling assay, evaluated Klotho protein expression by immunohistochemical assay, and assessed Nrf 2 and ARE protein expressions using western blotting assay. As compared with in the sham group, the SOD, MDA, and GSH concentrations were significantly deteriorated $(\mathrm{P}<0.001$, respectively); cardiomyocyte apoptosis index values were significantly increased $(\mathrm{P}<0.001)$; Klotho protein expression was significantly depressed; and Nrf-2 and ARE protein expressions were significantly $(\mathrm{P}<0.001$, respectively) in the model and pDC316 groups. However, with Klotho supplementation by pDC316 transfection, as compared with in the model group, the SOD, MDA, and GSH concentrations were significantly improved ( $\mathrm{P}<0.001$, respectively); the cardiomyocyte apoptosis index values were significantly suppressed $(\mathrm{P}<0.001)$; and the pathology was improved. Further, the Klotho protein expression of the pDC316-Klotho group was significantly upregulated and the Nrf-2 and ARE proteins expressions of the LY294002 and pDC316-Klotho groups were significantly suppressed. Klotho overexpression improved findings of oxidative stress injury after myocardial infarction.
\end{abstract}

Correspondence to: Dr Zhuofan Xu, Department of Internal Medicine-Cardiovascular, Guangzhou 12th People's Hospital, 1 Tianqiang Road, Guangzhou, Guangdong 510620, P.R. China E-mail: 76576831@qq.com

Key words: Klotho, oxidative stress, myocardial infarction, Nrf-2, ARE

\section{Introduction}

Myocardial infarction (MI) is known to be an important causative element in congestive heart failure. Persistent left ventricular (LV) remodeling after MI may lead to reduced LV systolic function and increase the incidence and mortality rates of cardiovascular events (1). Oxidative stress is a major cause of MI and, as such, the use of oxidants can effectively improve the disease progression (2). Kuro-o (2) was the first to successfully clone the a-Klotho gene (also known as the Klotho gene) in an aged mouse and found Klotho genes in both rats and humans that were highly homologous (83\%) with the mouse Klotho gene. The Klotho gene is located on chromosome 13 in humans, whereas the rat Klotho gene is on chromosome 12 , which is $50 \mathrm{~kb}$ in length and contains five exons. In the Klotho gene, its messenger RNA (mRNA) has an alternative splice site and, as such, it can produce membrane and secretory proteins. The human membrane Klotho protein is mainly expressed in the kidneys, small intestine and placenta; in effect, the human secretory Klotho protein essentially shares the same distribution pattern as the membrane Klotho protein. However, the secretory Klotho protein has no transmembrane or intracellular structure and functions primarily in the free form. Also, Klotho proteins can be found in the blood serum (3). Klotho gene mutations in mice are associated with relevant symptoms that resemble premature aging in humans and a shortened lifespan. In contrast, Klotho gene overexpression has been shown to extend the lifespan in mice (4). Relevant studies (5-7) demonstrated that downregulated Klotho expression under oxidative stress can result in greater damage to organs and tissues. However, the mechanism of action of the Klotho gene under MI-induced oxidative stress remains unclear. As such, the present study designed an MI model and injected Klotho gene-containing plasmids into the LV-free wall to investigate the effects of the Klotho gene on the MI model, and to explore its underlying mechanism by using the Nrf2/ARE pathway inhibitor LY294002 which could inhibit $\mathrm{Nrf} 2$ and ARE gene and protein expressions.

\section{Materials and methods}

Materials. Superoxide dismutase (SOD), glutathione (GSH), and malondialdehyde (MDA) assay kits (Nanjing Jiancheng Bioengineering Institute); Klotho, Nrf2, caspase-9 and ARE 
rabbit anti-mouse multiclonal antibodies (Abcam); recombinant adenovirus vector (Shanghai Shinegene Molecular Biotechnology); horseradish peroxidase (HRP)-labeled anti-mouse immunoglobulin G (ICL); a bicinchoninic acid protein assay kit (Shanghai Generay Biotech); a terminal deoxynucleotidyl transferase 2'-deoxyuridine-5'-triphosphate nick end-labeling (TUNEL) assay kit (Abcam); and an Nrf2/ARE signaling pathway inhibitor (LY2994002; Sigma-Aldrich, Merck KGaA) were employed. pDC316-NC and pDC316-Klotho were obtained from Nanjing KeyGEN Biotech Co., Ltd.

Modeling and grouping. Except for those in the sham group, each rat was injected with $45 \mathrm{mg} / \mathrm{kg}$ of pentobarbital, intubated, and connected to ventilators for assisted respiration, with the tidal volume set at $6.5 \mathrm{ml} / \mathrm{kg}$ and the respiratory frequency set at $100 \mathrm{breaths} / \mathrm{min}$. The left chest was shaved and the operative region was draped. An incision was made over the top of the fourth rib to open up the pleural cavity, expose the heart, and separate the pericardium. The left anterior descending coronary artery was ligated between the left atrium and the pulmonary artery with 5-0 Prolene (Ethicon, Inc.). During the operation, the MI region turned pale white. At the end of the operation, the chest walls were sutured layer by layer. Subsequently, the tracheal cannula was removed after the return of spontaneous respiration. The rat was not sent to a laboratory animal environment until it awoke from anesthesia. Two weeks after MI modeling, the study rats were divided into four groups-that is, the model, pDC316-NC, LY294002 and pDC316-Klotho groups. Intravenous tail injection was adopted, with the model, pDC316-NC, LY294002 and pDC316-Klotho groups injected with $200 \mu \mathrm{l}$ of normal saline, $200 \mu \mathrm{l}$ of pDC316 plasmids, $200 \mu \mathrm{l}$ of Klotho gene-containing plasmids, and $200 \mu \mathrm{l}$ of LY294002 $(15 \mathrm{mg} / \mathrm{kg})$, respectively. All groups were treated as mentioned above on a daily basis. The rats were fed regularly for six weeks and killed for histology after $12 \mathrm{~h}$ of fasting. The intra-abdominal injection of $3 \%$ pentobarbital $(45 \mathrm{mg} / \mathrm{kg})$ was performed before the rats were killed. Subsequently, half of the hearts of each group were stored at $-80^{\circ} \mathrm{C}$ and the other half were placed in $10 \%$ buffered formalin. This study received ethical approval from Guangzhou 12th People's Hospital.

Measurements of MDA, SOD, GSH and cardiac function in myocardial tissue. The rat myocardial tissue samples stored at $-80^{\circ} \mathrm{C}$ from each group were placed in a liquid nitrogen flash freezer and then ground into powder to measure the MDA, SOD and GSH levels in strict accordance with the instructions provided in the assay kit. The heart function of each group was evaluated by color Doppler echocardiography. The heart rate (HR), LV end-diastolic pressure (LVEDP), and LV systolic pressure (LVSP) were recorded.

Hematoxylin and eosin staining. At this stage, the heart tissues were removed from the $10 \%$ buffered formalin to dehydrate, clear and embed it into paraffin blocks. Subsequently, paraffin-embedded sections were prepared, measuring 3-4 $\mu \mathrm{m}$ in thickness, using a section cutter; dewaxed with xylene and stained for $15 \mathrm{~min}$ with a hematoxylin solution. Next, the sections were rinsed in running tap water; stained using blue hematoxylin for $30 \mathrm{sec}$ using Scott's Tap Water Substitute and then rinsed again in running tap water for $15 \mathrm{~min}$. Finally, the sections were stained with eosin, dehydrated with alcohol, cleared with xylene, mounted with neutral resin, analyzed with a light microscope, and captured the resultant images.

Masson's trichrome staining. After the routine paraffin section of myocardial tissue in the border area of MI was removed, Masson's trichrome staining was performed according to the instructions of the kit. The sections were stained with hematoxylin for $3 \mathrm{~min}$, washed with distilled water, differentiated with $1 \% \mathrm{HCL}-\mathrm{C}_{2} \mathrm{H}_{5} \mathrm{OH}$, returned to blue, soaked with fuchsin acid for three mins, differentiated with phosphomolybdic acid, redyed with aniline blue, and finally sealed by alcohol dehydration. Ten fields of vision were randomly selected for each section, and the myocardial collagen volume fraction (CVF) was measured using the Proplus 6.0 image analysis software (Media Cybernetics, Inc.).

TUNEL assay. The heart tissue was fixed in $10 \%$ buffered formalin, dehydrated, cleared and embedded into paraffin blocks; and subsequently adhered to poly-L-lysine-coated microscope slides. Following dewaxing; each slide was incubated for $60 \mathrm{~min}$ at $37^{\circ} \mathrm{C}$ in $50 \mu \mathrm{l}$ proteinase $\mathrm{K}$ solution. Finally, the sections were mounted with antifade mounting medium and the cells were counted under a fluorescence microscope. Those observed in blue were considered as normal myocardial cell nuclei, whereas apoptotic nuclei were stained dark brown. Images of the different target areas were captured with a light microscope in five vision fields (magnification, $\mathrm{x} 400$ ), in order to calculate the apoptotic index using the positive myocardial cells and the total myocardial cells in each visual field; that is, the proportion of apoptotic cells to the total myocardial cells in the same field of vision.

Assessing the expression of Klotho proteins in rat myocardial tissue by immunohistochemistry (IHC) assay. Antibodies in histiocytes were identified and located using a labeling developer and performed qualitative and quantitative analyses of the labeled antibodies, preparing and staining with relevant reagents according to the instructions given in the IHC assay kit. At this point, paraffin sections of muscular tissue were dewaxed twice using xylene, hydrated with alcohol step by step, and rinsed twice with phosphate-buffered saline (PBS). For deactivating endogenous enzymes, we incubated samples in a wet box with $3 \% \mathrm{H}_{2} \mathrm{O}_{2}$ for $10 \mathrm{~min}$ at room temperature. To complete antigen retrieval, a container filled with citrate was placed in a microwave oven, which was brought to a boil, and the slides were soaked in citrate for $3 \mathrm{~min}$, cooled to room temperature for renaturation, and rinsed twice with PBS. Subsequently, the slides were placed in the wet box, and normal goat serum was added and incubated for $10 \mathrm{~min}$ before removing it. Next, diluted primary Klotho antibody (dilution ratio, 1:200) was added to the wet box and incubated overnight at $4^{\circ} \mathrm{C}$. The following day, reagent 2 (a biotin-labeled secondary antibody) was added to incubate for $30 \mathrm{~min}$ at $37^{\circ} \mathrm{C}$, and then rinsed 3 times with PBS. HRP-labeled streptavidin was added to incubate for $10 \mathrm{~min}$ at room temperature, followed by three PBS rinses. Next, 3,3'-diaminobenzidine (DAB) was added and incubated for $5 \mathrm{~min}$ at room temperature. When cells presented with dark brown stain (positive) under observation, the development was stopped. The sections were then counterstained 
Table I. Primer sequences.

\begin{tabular}{ll}
\hline Gene name & \multicolumn{1}{c}{ Primer sequences } \\
\hline Klotho & F:5'-CTAGCTAGCCACCATGCCAGCCCGCGCCCCTCCTCGCC-3' \\
& R: 5'-ATTTGCGGCCGCTTATTTATAACGTCTCCGGCCTTTCT-3' \\
Nrf2 & F: 5'-TGGACGGGACTATTGAAGGCT-3' \\
& R: 5'-GCCGCCTTTTCAGTAGATGGA-3' \\
ARE & F: 5'-CACGCATATACCCGCTACC-3' \\
& R: 5'-AAGGCGGTCTTAGCCTCTTC-3' \\
Caspase-9 & F: 5'-GAGAGACATGCAGATATGGCATACA-3' \\
& R: 5'-CAGAAGTTCACGTTGTTGATGATG-3' \\
$\beta$-actin & F: 5'-CTGAGCCAGATGCTGTCCCATA-3' \\
& R: 5'-GAGACCATCCAAGGTCTCGATGTA-3'
\end{tabular}

F, forward; R, reverse.

with hematoxylin for 6 min, rinsed with running tap water, differentiated with hydrochloric acid for $1 \mathrm{sec}$, and dehydrated with alcohol. The slides were then cleared with xylene three times, mounted with gum, and observed using a microscope. Images were captured using a professional image acquisition and analysis system. Five different fields of vision were selected to observe each slice and the average greyscale of all positive results was analyzed.

Western blotting assay. Rat myocardial tissue from each experimental group was stored at $-80^{\circ} \mathrm{C}$. In order to obtain protein lysate, the tissue was ground thoroughly and the concoction was centrifuged to obtain the supernatant fluid. The protein concentration was measured using a bicinchoninic acid protein assay kit. A total of $20 \mu \mathrm{l}$ sample per lane was separated by sodium dodecyl sulfate-polyacrylamide gel electrophoresis (SDS-PAGE), and the proteins were transferred from the gel onto a cellulose filter, incubated with $5 \%$ skim milk solution for $1 \mathrm{~h}$ at room temperature. The membranes were then incubated in primary antibodies [dilution ratio: Klotho $=1: 2,000$; Nrf2=1:2,000; ARE=1:2,000; caspase-9=1:2,000; glyceraldehyde 3-phosphate dehydrogenase $(\mathrm{GAPDH})=1: 10,000]$ for overnight incubation at $4{ }^{\circ} \mathrm{C}$. This was followed by incubation with secondary antibody (dilution ratio $=1: 1,000$ ) for incubation for 2-3 $\mathrm{h}$ at room temperature. Finally, the membranes were treated with a developer and developed onto films. GAPDH was used as an internal control when the optical density of each band was quantified using a gel imaging system. The ratio of the optical density of each band to GAPDH was calculated, i.e., the result of the western blotting semiquantitative analysis of protein expression.

Reverse transcription polymerase chain reaction (RT-PCR) assay. The total RNA content was extracted from the tissues using TRIzol and all RNA were reverse-transcribed to complementary DNA (cDNA) using a reverse transcription kit (Takara Bio, Inc.). The mixture was centrifuged to the bottom with an instantaneous centrifuge and then put into a PCR apparatus. The reaction took place at $70^{\circ} \mathrm{C}$ for $10 \mathrm{~min}$ and the sample was quickly taken out and inserted onto ice to stop the reaction. The PCR reaction system was $20 \mu \mathrm{l}$ in total, consisting of $8 \mu \mathrm{l}$ PCR-level water, $10 \mu \mathrm{l} \mathrm{SYBR-Green} \mathrm{I}$ Master Mix, $0.5 \mu \mathrm{l}$ forward primer, $0.5 \mu \mathrm{l}$ reverse primer and $1 \mu \mathrm{l}$ cDNA template. The aforementioned reagents were mixed and were added into a 96-well plate that matched with the PCR instrument. Each sample was set with three multiple holes, centrifuged at 1,500 $\mathrm{g}$ for $3 \mathrm{~min}$, and tested on the PCR instrument. The primer sequence is shown in Table I.

Statistical analysis. All measurement data were expressed as means \pm standard deviations (SDs). An analysis of variance and Tukey's test were applied to the intergroup comparison of those following normal distribution and homogeneity of variance; in the case of non-normal distribution and heterogeneity of variance, the Kruskal-Wallis test by ranks was used, while the Nemenyi test was adopted for intergroup comparisons. $\mathrm{P}<0.05$ was used to indicate a statistically significant difference.

\section{Results}

MDA, SOD and GSH concentrations in the myocardial tissue. Compared with the sham group, the model group and the pDC316 group's SOD and GSH concentrations were significantly lower ( $\mathrm{P}<0.001$, respectively), whereas the MDA concentrations were markedly increased $(\mathrm{P}<0.001)$. On the hand, following treatment with LY294002 or pDC316-Klotho, the LY294002 and pDC316-Klotho groups exhibited a sharp increase in their SOD and GSH concentrations and a notable decline in MDA concentrations compared with the model group $(\mathrm{P}<0.001$, respectively). There was no significant difference noted in the SOD, MDA, or GSH concentrations between the model group and the pDC316 group ( $\mathrm{P}>0.05)$. Likewise, the differences between the LY294002 group and the pDC316-Klotho group in the SOD, MDA and GSH concentrations also lacked statistical significance $(\mathrm{P}>0.05)$. The data are shown in Fig. 1.

Cardiac function. Compared with the sham group, the LVSP was significantly downregulated and the HR and LVEDP were significantly upregulated in the model and pDC316 groups ( $\mathrm{P}<0.05$, respectively; Table II); however, with LY294002 and Klotho overexpression, the LVSP was significantly upregulated and the HR and LVEDP were significantly downregulated 
Table II. Comparison of cardiac function indexes of rats in each group (mean $\pm \mathrm{SD}$ ).

\begin{tabular}{lcccc}
\hline Group & $\mathrm{n}$ & LVSP $(\mathrm{mmHg})$ & LVEDP $(\mathrm{mmHg})$ & HR (time/min) \\
\hline Sham & 10 & $137.17 \pm 8.90$ & $7.88 \pm 0.93$ & $483.62 \pm 8.59$ \\
Model & 10 & $105.95 \pm 7.88^{\mathrm{a}}$ & $14.86 \pm 0.76^{\mathrm{a}}$ & $518.74 \pm 9.74^{\mathrm{a}}$ \\
PDC316 & 10 & $103.10 \pm 7.41^{\mathrm{a}}$ & $14.94 \pm 0.73^{\mathrm{a}}$ & $543.21 \pm 11.71^{\mathrm{a}}$ \\
LY294002 & 10 & $127.56 \pm 8.34^{\mathrm{b}}$ & $8.31 \pm 1.09^{\mathrm{b}}$ & $497.44 \pm 12.14^{\mathrm{b}}$ \\
pcDC316-Klotho & 10 & $128.07 \pm 8.92^{\mathrm{b}}$ & $8.86 \pm 1.31^{\mathrm{b}}$ & $500.57 \pm 14.93^{\mathrm{b}}$
\end{tabular}

${ }^{\mathrm{a}} \mathrm{P}<0.05$ compared with sham group; ${ }^{\mathrm{b}} \mathrm{P}<0.05$ compared with model group.
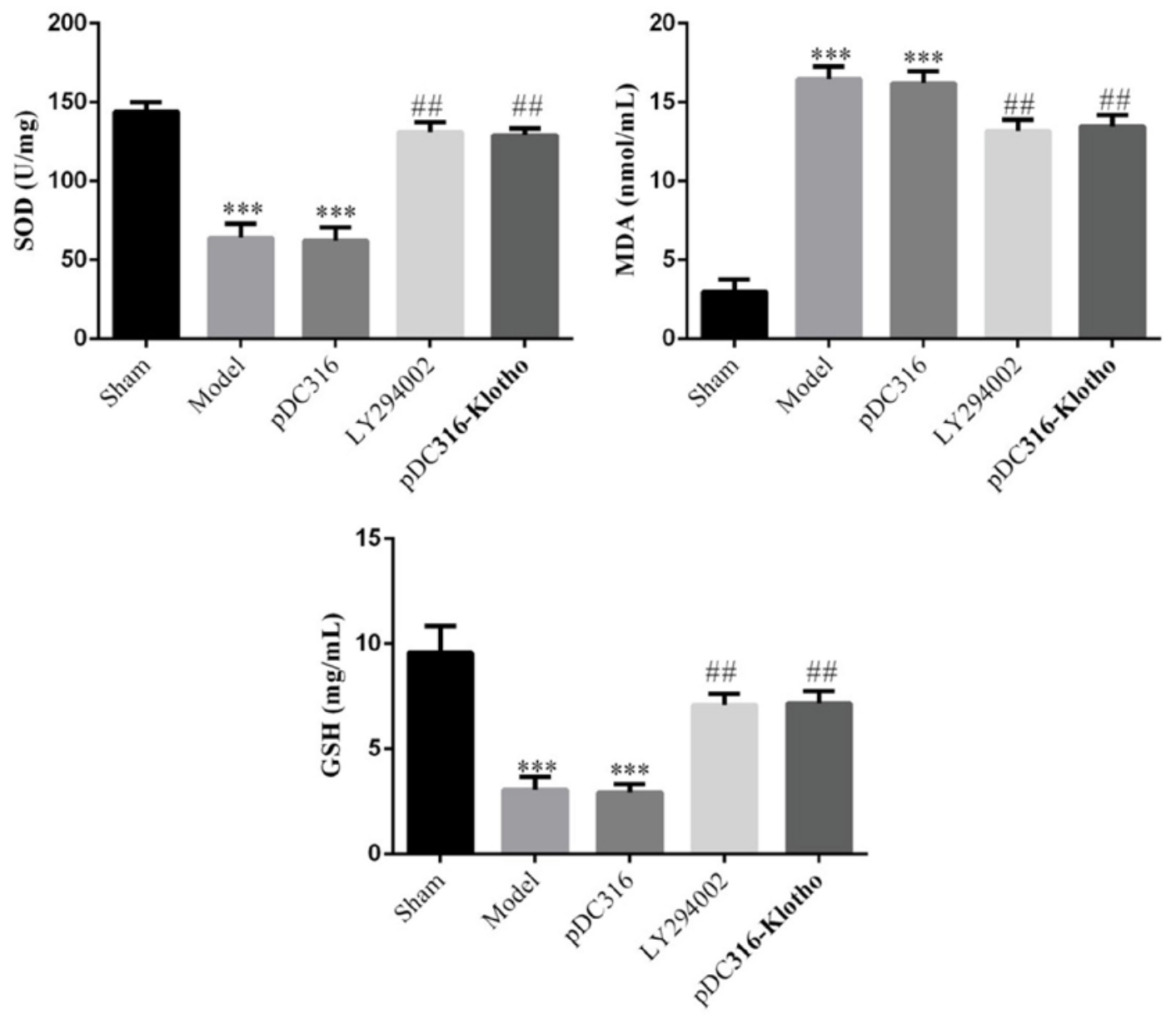

Figure 1. SOD, MDA and GSH concentration of different groups. Sham, sham group; model, myocardial infarction model group; pDC316, empty pDC316 were injected in rats based on myocardial infarction model group; LY294002, injection with LY294002 based on myocardial infarction model group; pDC316-Klotho, Klotho gene with pDC316 were injected in rats based on myocardial infarction model group. ${ }^{* * * *} \mathrm{P}<0.001 \mathrm{vs.} \mathrm{sham} \mathrm{group;}{ }^{\# \#} \mathrm{P}<0.01 \mathrm{vs}$. model group.

in the LY294002 and pDC316-Klotho groups $(\mathrm{P}<0.05$, respectively; Table II).

Myocardial pathology. Upon comparing the hematoxylin and eosin staining results of the sham group, myocardial necrosis, cytolysis, fragmentation. vascular engorgement, interstitial edema, increased infiltration of neutrophils, and stained cytoplasm were notable in the model and pDC316 groups (Fig. 2). Furthermore, compared with the model group, the LY294002 group and the pDC316-Klotho group both expressed reduced findings of myocardial necrosis, cytolysis, fragmentation, vascular engorgement, interstitial edema, increased infiltration of neutrophils, and stained cytoplasm (Fig. 2). Compared with the sham group, the $\mathrm{CVF} \%$ results of the model and pDC-316 groups were significantly increased; however, with LY294002, which inhibited the Nrf2/ARE pathway, and with Klotho overexpression, the CVF\% outcomes of the LY294002 and pDC316-Klotho groups were significantly decreased $(\mathrm{P}<0.001$, respectively; Fig. 3). These findings indicate that LY294002 and pDC316-Klotho can effectively resist pathological changes in rat hearts. 

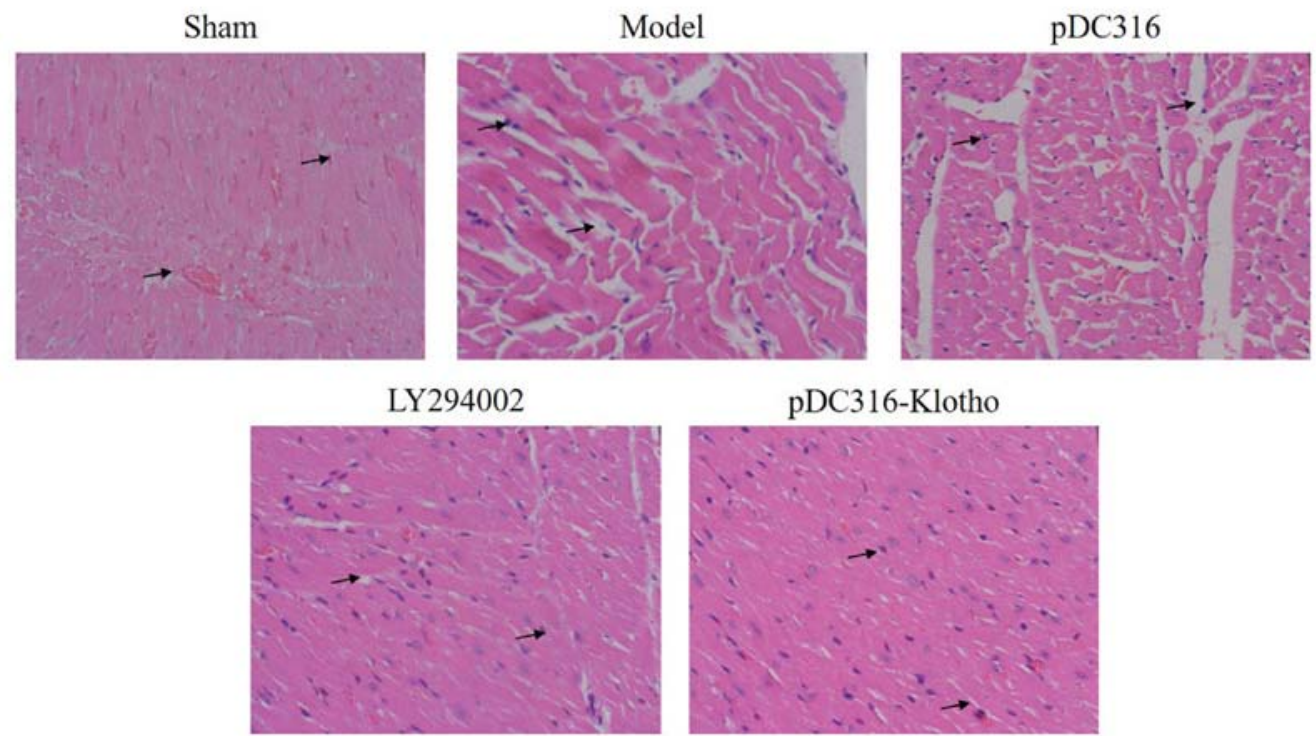

Figure 2. Pathology of different groups by H\&E staining (magnification, x200). Sham, sham group; model, myocardial infarction model group; pDC316, empty pDC316 were injected in rats based on myocardial infarction model group; LY294002, injection with LY294002 based on myocardial infarction model group; pDC316-Klotho, Klotho gene with pDC316 were injected in rats based on myocardial infarction model group.

Sham

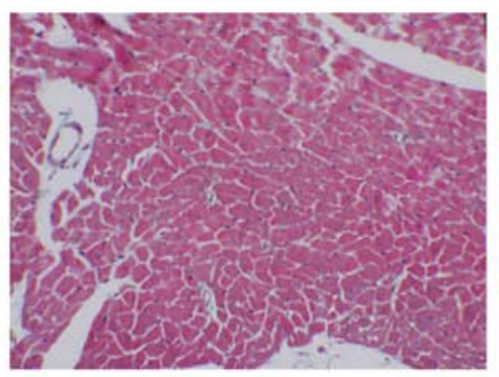

LY294002

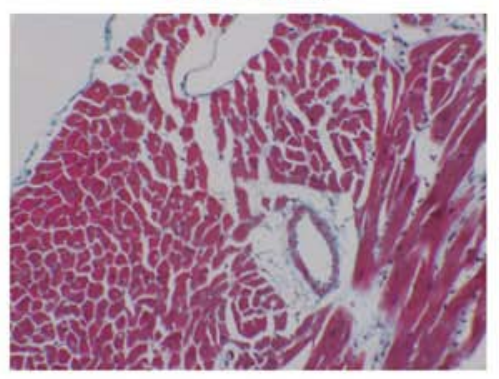

Model

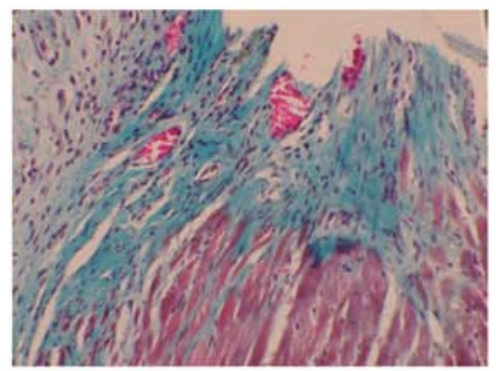

pDC316-Klotho

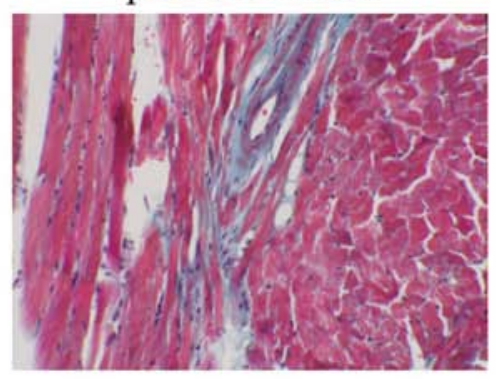

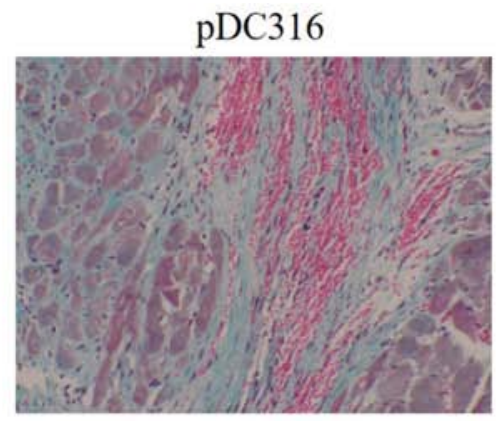

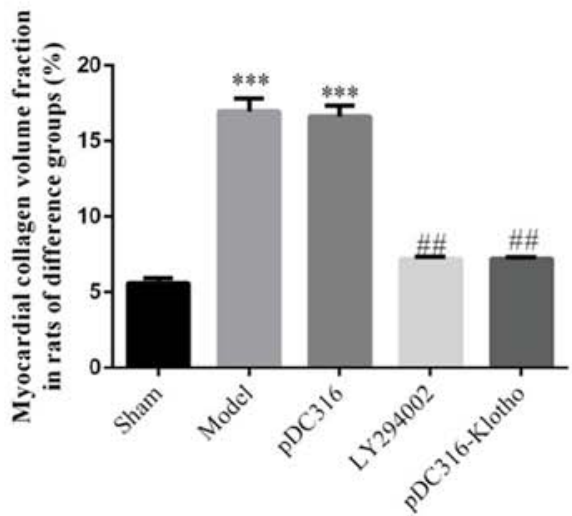

Figure 3. Myocardial collagen volume fraction in rats of different groups. Sham, sham group; model, myocardial infarction model group; pDC316, empty pDC316 were injected in rats based on myocardial infarction model group; LY294002, injection with LY294002 based on myocardial infarction model group; pDC316-Klotho, Klotho gene with pDC316 were injected in rats based on myocardial infarction model group. Magnification, $\mathrm{x} 200$. $^{* * *} \mathrm{P}<0.001 \mathrm{vs}$. sham group; ${ }^{\#} \mathrm{P}<0.01$ vs. model group.

Apoptosis myocardial tissue by TUNEL assay. Regarding microscopic findings, apoptotic nuclei were stained dark brown (Fig. 3). Compared with the sham group, the model group and the pDC316 group presented with much higher apoptotic indexes $(\mathrm{P}<0.001$, respectively; Fig. 4). Following intervention with LY294002 or pDC316-Klotho, the
LY294002 group and the pDC316-Klotho group's apoptotic indexes were significantly lower than those of the model group $(\mathrm{P}<0.01$, respectively; Fig. 4). However, the intergroup difference (model vs. pDC316; LY294002 vs. pDC316-Klotho) in terms of apoptotic index was not statistically significant $(\mathrm{P}>0.05)$. 


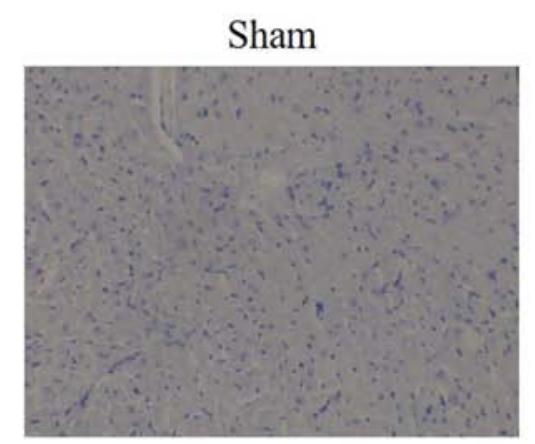

LY294002

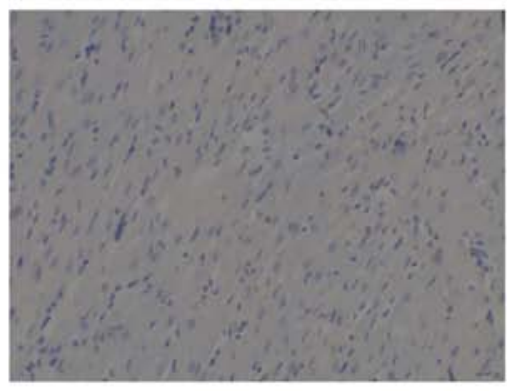

Model

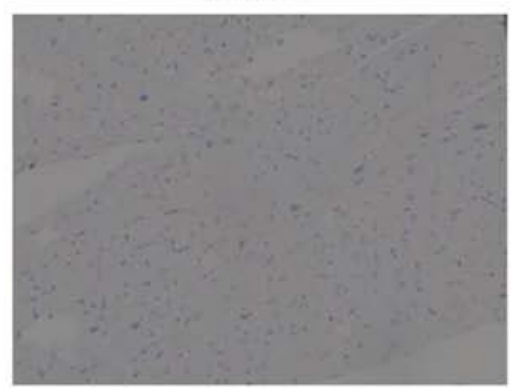

pDC316-Klotho

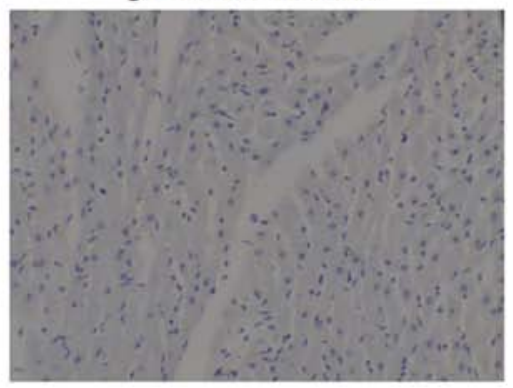

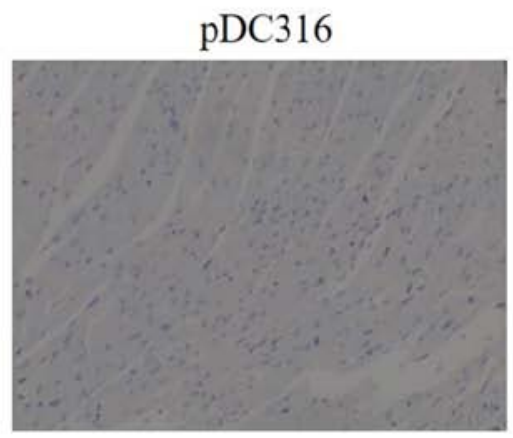

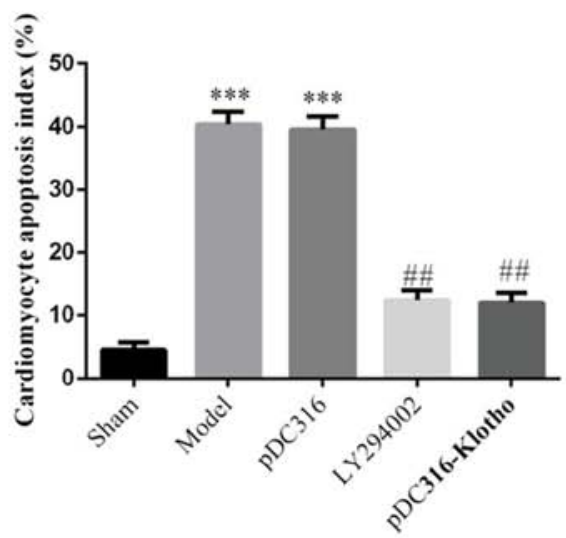

Figure 4. Cardiomyocyte apoptosis index of different groups determined by TUNEL assay. Sham, sham group; model, myocardial infarction model group; pDC316, empty pDC316 were injected in rats based on myocardial infarction model group; LY294002, injection with LY294002 based on myocardial infarction model group; pDC316-Klotho, Klotho gene with pDC316 were injected in rats based on myocardial infarction model group. Magnification, $\mathrm{x} 200 .{ }^{* * *} \mathrm{P}<0.001$ vs. sham group; ${ }^{\#} \mathrm{P}<0.01$ vs. model group.

Expression of Klotho proteins in myocardial tissue. The IHC assay indicated that the expression of Klotho proteins of the model, pDC316, and LY294002 groups were significantly lower than in the sham group $(\mathrm{P}<0.001$, respectively; Fig. 4). However, these three groups showed no significant inter-group differences $(\mathrm{P}>0.05)$. Compared with the model group, the pDC316-Klotho group had a significantly elevated level of Klotho expression $(\mathrm{P}<0.01)$. The relative data are shown in Fig. 5.

Klotho, Nrf2, ARE and caspase-9 mRNA and protein expressions in each group's myocardial tissue by RT-PCR and western blotting assay. According to the western blotting and RT-PCR assay results, the model group and the pDC316 group showed higher expression of Nrf2, ARE and caspase-9 compared with the sham group $(\mathrm{P}<0.001$, respectively; Fig. 6A and B); however, there was no significant difference between the model group and the pDC316 group ( $\mathrm{P}>0.05)$. Following LY294002 and pDC316-Klotho treatment, the Nrf2, ARE and caspase-9 expression levels of the LY294002 group and the pDC316-Klotho group were clearly inhibited in comparison with those of the model group $(\mathrm{P}<0.01$, respectively; Fig. $6 \mathrm{~A}$ and $\mathrm{B})$. Furthermore, compared with in the sham group, the Klotho gene and protein expression levels of the model, pDC316 and LY294002 groups were significantly decreased $(\mathrm{P}<0.001$, respectively; Fig. 6A and B). Finally, with Klotho overexpression, the Klotho gene and protein expression levels of the pcDC316-Klotho group were significantly increased compared with the model group.

\section{Discussion}

MI can cause ischemia, hypoxia and ischemia-reperfusion injury of local tissue, and local or systemic oxidative stress when a large number of oxygen free radicals are produced by NADPH oxidase, xanthine oxidase, and respiratory chains in neutrophils through oxygen bursts. Further, oxygen free radicals may result in damage to myocardial cells and induce apoptosis and necrosis using the nuclear factor kappa B signaling pathway that $\mathrm{c}-\mathrm{Jun} \mathrm{N}$-terminal kinase heavily depends upon, or cause MI-associated pathological damage to the myocardial tissue through other pathways (8-10). Antioxidants can effectively inhibit post-MI oxidative stress, thereby improving cardiac function and promoting heart repair. Zhou et al (11) found that probucol, an antioxidant, could effectively inhibit the level of post-MI oxidative stress and collagen remodeling and improve the diastolic function of the left ventricle. Khanna et al (12) reported that smoking was associated with a larger MI-related zone and LV hypofunction, accompanied by increased proinflammatory factors, tissue repair factors, and oxidative stress markers in the myocardial tissue; the use of another antioxidant $\mathrm{N}$-acetylcysteine resulted in a smaller MI-related zone, improved cardiac function, and a significantly lower level of proinflammatory factors and oxidative stress markers 


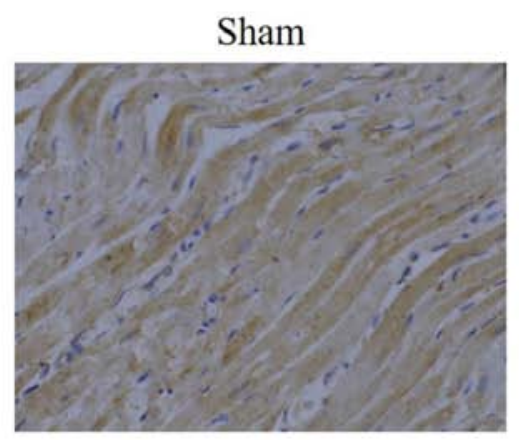

LY294002

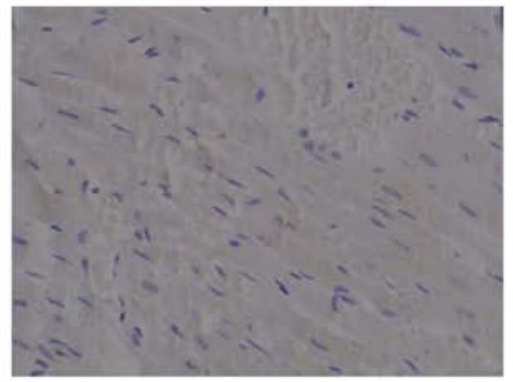

Model

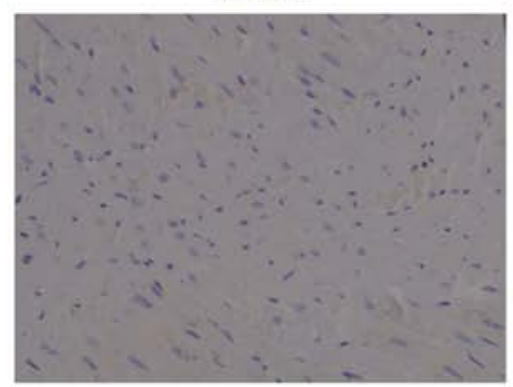

pDC316-Klotho

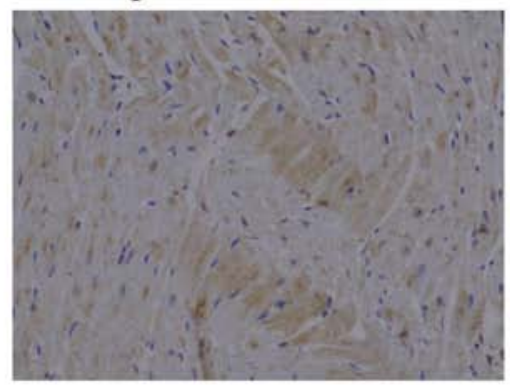

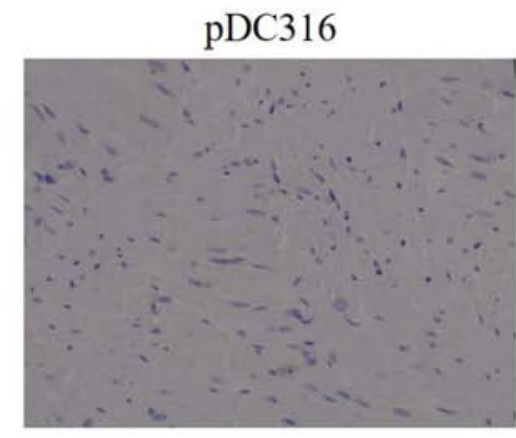

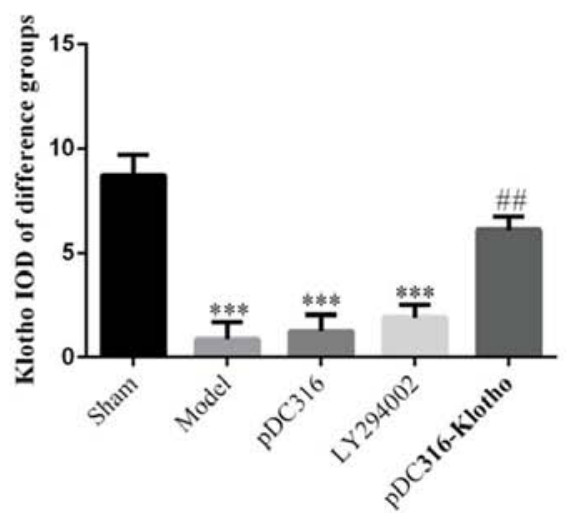

Figure 5. Klotho protein expression of different groups determined by immunohistochemistry assay. Sham, sham group; model, myocardial infarction model group; pDC316, empty pDC316 were injected in rats based on myocardial infarction model group; LY294002, injection with LY294002 based on myocardial infarction model group; pDC316-Klotho, Klotho gene with pDC316 were injected in rats based on myocardial infarction model group. Magnification, x200. ${ }^{* * *} \mathrm{P}<0.001$ vs. sham group; ${ }^{\#} \mathrm{P}<0.01$ vs. model group.

in the myocardial tissue, which is likely correlated with the substantial increase in the transcription levels of SOD, thioredoxin, Nrf2 and the circulating GSH. In the present study, the SOD and GSH concentrations of the model group increased significantly, whereas this group's MDA concentration dropped sharply. This outcome suggests the activation of MI-induced oxidative stress. However, following intervention with LY294002 (Nrf2/ARE signaling pathway inhibitor) and pDC316-Klotho, the oxidative stress level of the myocardial tissue was markedly inhibited. Also, hematoxylin and eosin and TUNEL staining provided evidence for the myocardial damage at a remarkably and increasingly severe level, which was improved substantially by LY294002 Nrf2/ARE signaling pathway inhibition and pDC316-Klotho.

Despite the regulation by multiple factors, recent studies have noticed Klotho's extensive physiological and pathophysiological functions and its presence in the development and progression of many diseases. The Klotho gene is primarily expressed in the kidneys, small intestine, lungs, prostate, blood and brain tissue. The Klotho gene acts as an antioxidant agent by improving the expression of such antioxidant genes, such as catalases and SOD (13). Based on the aforementioned studies, this study observed that the Klotho expression of the model group was significantly lower than that of the high-fat-fed control group. When the Klotho proteins play a role of antioxidation, oxidative stress can downregulate the Klotho expression through a range of pathways. Some studies $(14,15)$ have suggested that Klotho proteins were dramatically reduced in the presence of diabetes, hypertension, and renal diseases, whereas oxidative stress had an important impact on the development and progression of these diseases. The expression of Klotho proteins in the myocardial tissue was significantly increased following pDC316-Klotho treatment, which may explain the post-MI alleviation of apoptosis. On the other hand, LY294002, an inhibitor of the Nrf2/ARE signaling pathway, also demonstrated the ability to repair damage to the myocardial tissue.

With Nrf 2 being an important transcription factor that reduces reactive oxygen in oxidative stress, the $\mathrm{Nrf} 2 / \mathrm{ARE}$ signaling pathway plays a crucial role in oxidative stress. Normally, Nrf2 remains deactivated in the cytoplasm and thus cannot enter the cell nuclei as a transcription factor. However, Nrf2 will be activated when exposed to oxidative stress. The activated Nrf2 enters the cell nuclei and forms heterodimers with small Maf proteins. Together with ARE, the expression of downstream target genes is promoted to mediate a series of reactions to produce MnSOD (Manganese superoxide dismutase), HO-1 (heme oxygenase-1) and other enzymes, thereby improving the systemic antioxidant ability $(16,17)$. When oxidation-reduction reactions reach equilibrium, Nrf2 returns to the cytoplasm and maintains a normal level through degradation or negative feedback, via the ubiquitin-proteasome pathway (18). The results of the present study indicate that both LY294002 (Nrf2/ARE signaling pathway inhibitor) and pDC316-Klotho can effectively impede the Nrf2/ARE signaling pathway and reduce damage to the myocardial tissue caused by post-MI oxidative stress. 
A
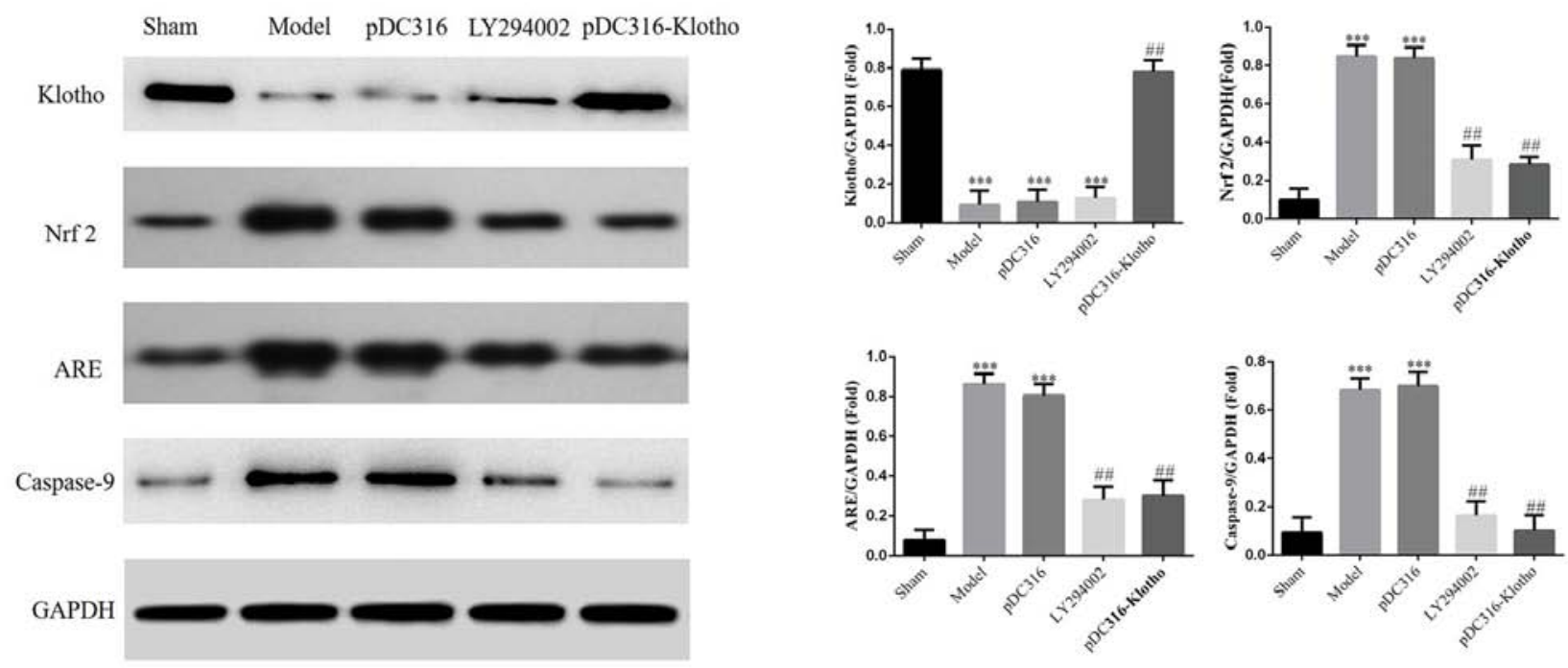

B
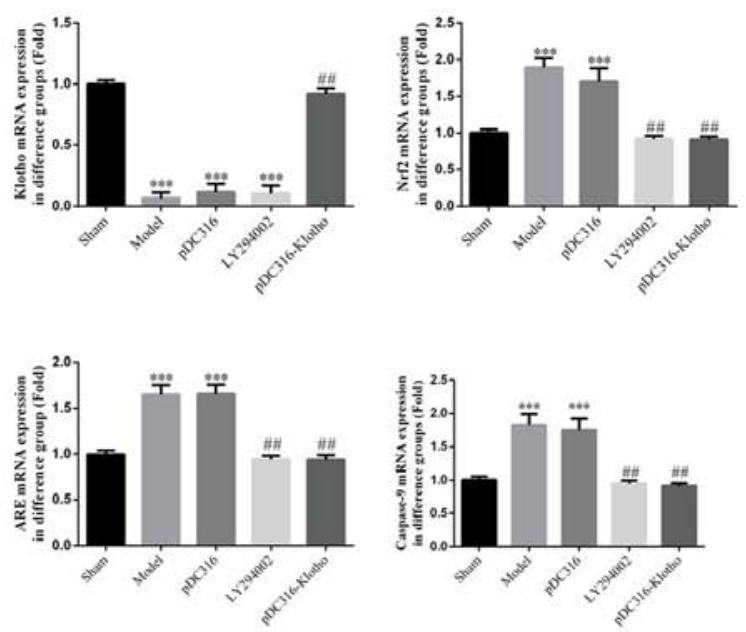

Figure 6. Klotho, Nrf 2, ARE and caspase-9 protein and mRNA expression levels of different groups determined by WB and RT-PCR assay. (A) Relative protein expression of different groups by WB assay. (B) Relative mRNA expression of different groups by RT-PCR assay. Sham, sham group; model, myocardial infarction model group; pDC316, empty pDC316 were injected in rats based on myocardial infarction model group; LY294002, injection with LY294002 based on myocardial infarction model group; pDC316-Klotho, Klotho gene with pDC316 were injected in rats based on myocardial infarction model group. ${ }^{* * * *} \mathrm{P}<0.001$ vs. sham group; ${ }^{\# \#} \mathrm{P}<0.01$ vs. model group. WB, western blotting; RT-PCR, reverse transcription-PCR.

In summary, overexpression of the Klotho gene, to a certain degree, can repair damage to myocardial tissue caused by post-MI oxidative stress through inhibiting the Nrf2/ARE signaling pathway.

\section{Acknowledgements}

Not applicable.

\section{Funding}

No funding was received.

\section{Availability of data and materials}

The datasets used and/or analyzed during the current study are available from the corresponding author on reasonable request.

\section{Authors' contributions}

ZX conceptualized and developed the study design, and performed the majority of the experiments. SZ and XF acquired the data, which were analyzed by SZ, XF, CC, XY and PL. XY and PL wrote the manuscript, and XF and CC suggested appropriate modifications, which were corrected by SZ. All authors read and approved the final manuscript.

\section{Ethics approval and consent to participate}

This study received ethical approval from Guangzhou 12th People's Hospital.

\section{Patient consent for publication}

Not applicable. 


\section{Competing interests}

The authors declare that they have no competing interests.

\section{References}

1. Liu X, Hou L, Xu D, Chen A, Yang L, Zhuang Y, Xu Y, Fassett JT and Chen Y: Effect of asymmetric dimethylarginine (ADMA) on heart failture development. Nitric Oxide 54: 73-81, 2016.

2. LeBaron TW, Kura B, Kalocayova B, Tribulova N and Slezak J: A new approach for the prevention and treatment of cardiovascular disorders. Molecular hydrogen significantly reduces the effects of oxidative stress. Molecules 24: pii: E2076, 2019.

3. Kuro-o M, Matsumura Y, Aizawa H, Kawaguchi H, Suga T, Utsugi T, Ohyama Y, Kurabayashi M, Kaname T, Kume E, et al Mutation of the mouse klotho gene leads to a syndrome resembling ageing. Nature 390: 45-51, 1997.

4. Zhou HJ, Zeng CY, Yang TT, Long FY, Kuang X and Du JR Lentivirus-mediated klotho up-regulation improves aging-related memory deficits and oxidative stress in senescence-accelerated mouse prone-8 mice. Life Sci 200: 56-62, 2018.

5. Mitobe M, Yoshida T, Sugiura H, Shirota S, Tsuchiya K and Nihei H: Oxidative stress decreases klotho expression in a mouse kidney cell line. Nephron Exp Nephrol 101: e67-e74, 2005.

6. Padanilam BJ: Cell death induced by acute renal injury: A perspective on the contributions of apoptosis and necrosis. Am J Physiol Renal Physiol 284: F608-F627, 2003.

7. Yamamoto M, Clark JD, Pastor JV, Gurnani P, Nandi A, Kurosu H, Miyoshi M, Ogawa Y, Castrillon DH, Rosenblatt KP and Kuro-o M: Regulation of oxidative stress by the anti-aging hormone klotho. J Biol Chem 280: 38029-38034, 2005.

8. Bagatini MD, Martins CC, Battisti V, Gasparetto D, da Rosa CS, Spanevello RM, Ahmed M, Schmatz R, Schetinger MR and Morsch VM: Oxidative stress versus antioxidant defenses in patients with acute myocardial infarction. Heart Vessels 26: 55-63, 2011.

9. Kuroda J, Ago T, Matsushima S, Zhai P, Schneider MD and Sadoshima J: NADPH oxidase 4 (Nox4) is a major source of oxidative stress in the failing heart. Proc Natl Acad Sci USA 107: 15565-15570, 2010.
10. Williams AR and Hare JM: Mesenchymal stem cells: Biology, pathophysiology, translational findings, and therapeutic implications for cardiac disease. Circ Res 109: 923-940, 2011.

11. Zhou SX, Zhou Y, Zhang YL, Lei J and Wang JF: Antioxidant probucol attenuates myocardial oxidative stress and collagen expressions in post-myocardial infarction rats. J Cardiovasc Pharmacol 54: 154-162, 2009.

12. Khanna AK, Xu J and Mehra MR: Antioxidant N-acetyl cysteine reverses cigarette smoke-induced myocardial infarction by inhibiting inflammation and oxidative stress in a rat model. Lab Invest 92: 224-235, 2012.

13. Olejnik A, Franczak A, Krzywonos-Zawadzka A, Kałużna-Oleksy M and Bil-Lula I: The biological role of Klotho protein in the development of cardiovascular diseases. Biomed Res Int 2018: 5171945, 2018.

14. Hu MC, Kuro-o M and Moe OW: Renal and extrarenal actions of Klotho. Semin Nephrol 33: 118-129, 2013.

15. Liu JJ, Liu S, Morgenthaler NG, Wong MD, Tavintharan S, Sum CF and Lim SC: Association of plasma soluble $\alpha$-klotho with pro-endothelin-1 in patients with type 2 diabetes. Atherosclerosis 233: 415-418, 2014.

16. Menshchikova EB, Zenkov NK, Tkachev VO, Lemza AE and Kandalintseva NV: Protective effect of ARE-inducing phenol antioxidant TS-13 in chronic inflammation. Bull Exp Biol Med 155: 330-334, 2013.

17. Jing $X$, Ren D, Wei $X$, Shi H, Zhang X, Perez RG, Lou H and Lou H: Eriodictyol-7-O-glucoside activates Nrf2 and protects against cerebral ischemic injury. Toxicol Appl Pharmacol 273: 672-679, 2013.

18. Canning P, Cooper CD, Krojer T, Murray JW, Pike AC, Chaikuad A, Keates T, Thangaratnarajah C, Hojzan V, Ayinampudi V, et al: Structural basis for Cul3 protein assembly with the BTB-Kelch family of E3 ubiquitin ligases. J Biol Chem 288: 7803-7814, 2013.

This work is licensed under a Creative Commons Attribution-NonCommercial-NoDerivatives 4.0 International (CC BY-NC-ND 4.0) License. 